\title{
Effect of entanglement on dual condensates at finite isospin chemical potential from an entangled Polyakov-Nambu- Jona-Lasinio model
}

\author{
Zhao Zhang ${ }^{1, a}$ and Qing Miao ${ }^{1}$ \\ ${ }^{1}$ School of Mathematics and Physics, North China Electric Power University, Beijing 102206, China
}

\begin{abstract}
The dual observables as order parameters for deconfinement transition are investigated at finite isospin chemical potential $\mu_{I}$ in an entangled Polyakov-Nambu-Jona-Lasinio model of QCD. Compared to PNJL, the four quark vertice with entanglement between the Polyakov loop and the chiral and pion condensates are considered in this model. We mainly concentrate on the role of this entanglement on the thermal behavior of the dressed polyakov loop and the dual pion condensate for $\mu_{I}>m_{\pi} / 2$. We find that the $T$-dependences of these dual condensates are qualitatively consistent with the PNJL results. Due to the entanglement, the critical temperatures determined by the quark and pion condensates and their corresponding dual partners get close to that extracted from the Polyakov loop.
\end{abstract}

\section{Introduction}

The main phenomena in QCD at finite temperature and density are the chiral restoration and deconfining phase transitions. In the chiral limit, the standard order parameter for chiral transition is the quark condensate. However, it is conceptually difficult to define an order parameter for deconfinement in QCD. Usually, the expectation value of the Polyakov loop (PL) is used to indicate the quark deconfining transition. This quantity is a true order parameter for center symmetry in pure Yang-Mills theory. Nevertheless, this symmetry is badly broken by the light dynamical quarks in QCD. These two (effective) order parameters had been extensively studied in lattice QCD at finite $T$ and zero density and it suggests that two pseudo critical temperatures are very close to each other [1,2].

Recently, some dual observables are introduced as the new order parameters for center symmetry by using the twisted boundary conditions for quarks [3-6]. Especially, it is demonstrated in the formalism of lattice QCD [3, 4] that the dressed Polyakov loop (DPL) interpolates between the chiral condensate and the thin PL. The studies from the functional methods [7-10] and effective models [11-13] also suggest the DPL shows the order parameterlike behavior like the thin PL. In principle, one can construct many dual observables which transform in the same way as the thin PL under the center transformation $[5,6]$. They are true order parameters for deconfinement in the heavy quark limit $m \rightarrow \infty$.

ae-mail: zhaozhang@pku.edu.cn 
However, the center symmetry is seriously broken in QCD since the light quark masses are very small. Then, a question naturally arises: to what extent do these quantities still contain the confinement information? Recently, it is demonstrated in the NJL model that the rapid rise of DPL near $T_{c}$ is totally driven by the chiral transition [14]. The following study based on several variants of NJL with (possible) confining elements suggests that the rapid rise of DPL with $T$ still happens in the chiral transition region and no effect related to the change of the confining properties of the propagator is observed [15]. The authors thus conclude that the DPL obtained in these models is not an appropriate order parameter for deconfinement. In addition, the test of dual observables as order parameters has been extended to finite isospin chemical potential by considering the pion condensation [16]. It is found in PNJL that not only the dressed Polyakov loop, but also the dual pion condensate (DPC) are quite sensitive to the chiral dynamics and pion condensation rather than the thin PL [16]. Thus it may be difficult to distinguish the deconfinement transition from the dual observables, at least in the NJL-type models.

We stress that the PNJL results in [16] may not really true in QCD. In other words, the possible subtle relationship between the center symmetry breaking and chiral transition existing in QCD may not be totally captured by PNJL, even the center symmetry is considered in this model. Note that it derived in Ref.[17] that a particular non-local version of PNJL can re regarded as a low energy limit of QCD. Inspired by this work, the entangled PNJL (EPNJL) model has been proposed [18]. Compared to PNJL, the four-quark interaction is dependent on the Polyakov-loop in EPNJL. So the coupling or entanglement between the PL and quark condensate in EPNJL is somewhat stronger than that in PNJL. The motivation of this paper is to investigate the role of such an enhanced entanglement on the thermal properties of the dual condensates at finite isospin chemical potential for $\mu_{I}>m_{\pi} / 2$.

\section{EPNJL model at finite isospin chemical potential with twisted boundary conditions}

\subsection{Two flavor EPNJL model and the model parameters}

We adopt the following lagrangian of two-flavor EPNJL model

$$
\begin{aligned}
\mathcal{L}= & \bar{\psi}\left(i \gamma_{\mu} D^{\mu}+\gamma_{0} \hat{\mu}-\hat{m}_{0}-i \lambda \gamma_{5} \tau_{1}\right) \psi \\
& +g_{s}(\Phi)\left[(\bar{\psi} \psi)^{2}+\left(\bar{\psi} i \gamma_{5} \vec{\tau} \psi\right)^{2}\right]-g_{v}^{s}\left(\bar{\psi} \gamma_{\mu} \psi\right)^{2} \\
& -g_{v}^{v}\left(\bar{\psi} \vec{\tau} \gamma_{\mu} \psi\right)^{2}-\mathcal{U}(\Phi, \bar{\Phi}, T)
\end{aligned}
$$

which is an improved version of PNJL used in [16]. The last term in (1) is the pure gauge sector described by the Polyakov loop $\Phi$, which is invariant under the $Z(3)$ transformation. As mentioned, the scalar coupling $g_{s}$ is explicitly $\Phi$ dependent in this model. According to the suggestion in [18], the form of $g_{s}$ below is employed in this paper:

$$
g_{s}(\Phi)=g_{s}\left[1-\alpha_{1} \Phi \bar{\Phi}-\alpha_{2}\left(\Phi^{3}+\bar{\Phi}^{3}\right)\right]
$$

which respects the chiral, $\mathrm{P}, \mathrm{C}$ and the extended $Z(3)$ symmetries. Following [18], both $\alpha_{1}$ and $\alpha_{2}$ are fixed as 0.2 which can reproduce the available lattice data. The model parameters other than $g_{s}(\Phi)$ in (1) are all adopted from [16] in this paper. Note that $g_{s}$ in (2) is also the same as that in [16]. The possible $\Phi$-dependence of $g_{v}^{v(s)}$ is ignored in our calculation. 


\subsection{EPNJL with twisted boundary conditions for $\mu_{I}>m_{\pi} / 2$}

It is well known that the charged pion condensation appears for zero and low temperatures for $\mu_{I}>m_{\pi} / 2$ [19], where $\mu_{I}$ is the isospin chemical potential. The study based on PNJL in [20] suggests that relation between the PL and pion condensate at finite temperature for $\mu_{I}>m_{\pi} / 2$ is quite similar to that between the PL and quark condensate at zero isospin density. In addition, the test of the dual condensates as order parameters for center symmetry has also been extended to finite isospin chemical potential in [16], where the PNJL model is adopted. Besides the extensively studied DPL, another simple dual observable, namely the dual pion condensate is proposed and studied in [16]. These two dual condensates are defined as

$$
\Sigma_{\sigma}^{(1)}=-\int_{0}^{2 \pi} \frac{d \phi}{2 \pi} e^{-i \phi} \sigma(\phi),
$$

and

$$
\Sigma_{\pi}^{(1)}=-\int_{0}^{2 \pi} \frac{d \phi}{2 \pi} e^{-i \phi} \pi(\phi),
$$

respectively. Note that $\sigma(\phi)(\pi(\phi))$ is the generalized quark (pion) condensate for the twisted angle $\phi$.

When considering the $\Phi$-dependence of $g_{s}$, the two energy gaps in [16] are modified as

$$
M=m-2 g_{s}(\Phi) \sigma(\phi), \quad N=\lambda-2 g_{s}(\Phi) \pi(\phi) .
$$

We still use $\mu^{\prime}$ and $\mu_{I}^{\prime}$ to indicate the shifted quark and isospin chemical potentials when considering the vector interaction

$$
\mu^{\prime}=\mu-2 g_{v}^{s}\left(\rho_{u}+\rho_{d}\right), \quad \mu_{I}^{\prime}=\mu_{I}-2 g_{v}^{v}\left(\rho_{u}-\rho_{d}\right),
$$

where $\rho_{u(d)}$ is the $\mathrm{u}(\mathrm{d})$ quark density. The thermal potential of EPNJL at the mean field level still takes the form of Eq.(14) in [16].

Under the generalized boundary condition, the modified quark chemical potential $\mu^{\prime}$ in the thermal potential should be replaced by $i T(\phi-\pi)[3,6,11]$, which is nothing but an effective imaginary chemical potential. Strictly speaking, the $\mu^{\prime}$ for $\phi \neq \pi$ should also contain the density-related contribution $2 g_{v}^{s}\left(\rho_{u}+\rho_{d}\right)$ even the real $\mu$ is zero. This is because the imaginary chemical potential also leads to a nonzero baryon number density. It has been shown in [11] that the coupling $g_{v}^{s}$ only has significant effect on $\Sigma_{\sigma}^{(1)}$ for $T>1.5 T_{c}$ in PNJL. Since we are only interested in the thermal behavior of dual observables near and below $T_{c}$, the correction $2 g_{v}^{s}\left(\rho_{u}+\rho_{d}\right)$ is ignored in our calculation. Note that the $\mu_{I}^{\prime}$ is still real and keeps the form as (6). As in [16], we only conduct the calculation for the case with $\mu_{I}>m_{\pi} / 2$ and zero $\mu$.

\section{Results and discussion}

\section{$3.1 \phi$-dependence of quark and pion condensates}

The generalized quark condensate as function of $\phi$ for $\mu_{I}=100 \mathrm{MeV}$ at different temperatures is shown in Fig. 1. We see that the shapes of $|\sigma(\phi)|$ for temperatures below and above $T_{c}^{\chi}$ are quite different: for $T=190 \mathrm{MeV}$ and $230 \mathrm{MeV}$ (or $T>T_{c}^{\chi}$ ), the quark condensates are concave lines with $|\sigma(\pi)|<|\sigma(0)|$; but for $T=150 \mathrm{MeV}$ and $170 \mathrm{MeV}$ (or $T<T_{c}^{\chi}$ ), they are 


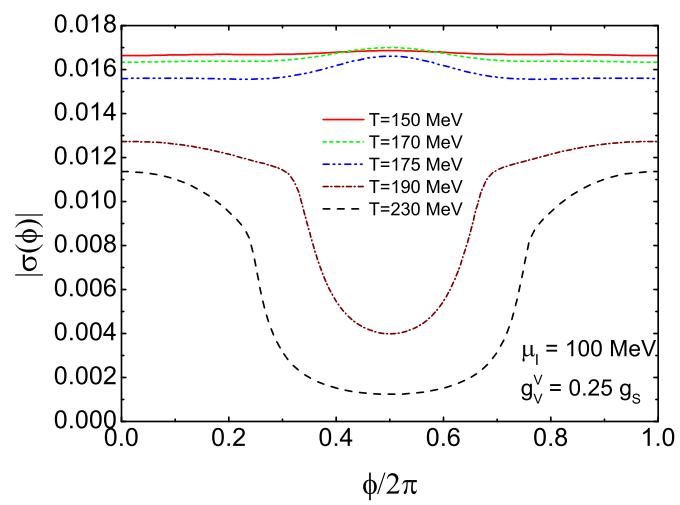

Figure 1. The twisted angle dependence of the quark condensate $\sigma(\phi)$ at $\mu_{I}=100 \mathrm{MeV}$ for different temperatures

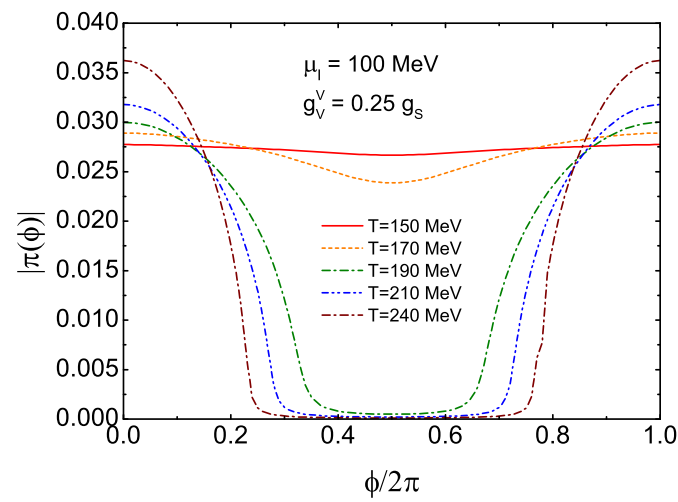

Figure 2. The twisted angle dependence of the pion condensate $\pi(\phi)$ at $\mu_{I}=100 \mathrm{MeV}$ for different temperatures

convex ones with $|\sigma(\pi)|>|\sigma(0)|$. All this is very similar to Fig.1.a in [16] but quite different from what obtained in $[3,8,11]$, where only concave curves emerge for vanishing $\mu$ and $\mu_{I}$.

The generalized pion condensate as function of $\phi$ for $\mu_{I}=100 \mathrm{MeV}$ at different temperatures is shown in Fig. 2. In contrast, Fig. 2 shows that all lines of $|\pi(\phi)|$ at different fixed $T$ are concave curves. We see that in the fermionic-like region (namely the area for $\phi \sim \pi$ ), $|\pi(\phi)|$ decreases with $T$ but it increases with $T$ in the bosonic-like region (namely the area for $\phi$ near zero or $2 \pi$ ). In addition, the curve of $|\pi(\phi)|$ becomes more flat with decreasing $T$. All those is very similar to the $\phi$-dependence of the quark condensate obtained for zero $\mu$ and $\mu_{I}[3,8,11]$, which is qualitatively consistent with the PNJL result, namely Fig.1.b in [16]. 


\subsection{Thermal behaviors of dual condensates for $\mu_{I}>m_{\pi} / 2$}

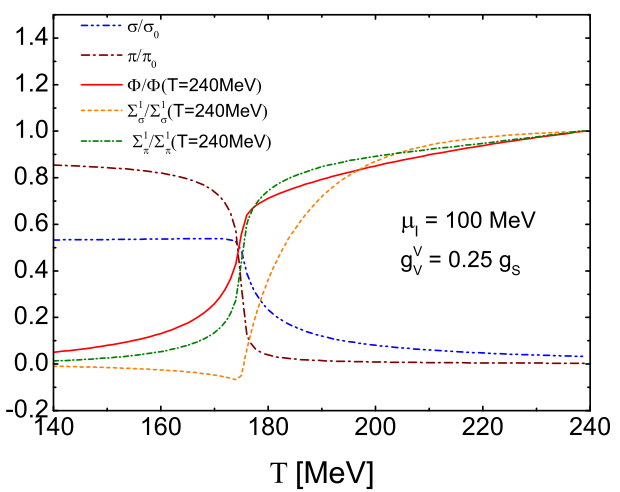

Figure 3. The temperature dependence of the normalized conventional ployakov loop, quark and pion condensates and their corresponding dual parters at $\mu_{I}=100 \mathrm{MeV}$.

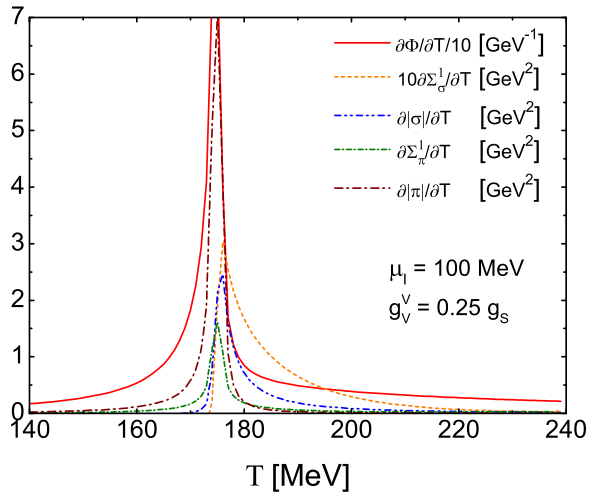

Figure 4. The temperature dependence of the T-derivatives of the conventional ployakov loop, quark and pion condensates and their corresponding dual parters at $\mu_{I}=100 \mathrm{MeV}$.

Two dual condensates and other three (pseudo-) order parameters as functions of $T$ for $\mu_{I}=100 \mathrm{MeV}$ are shown in Figs. 3. The quark and pion condensates are obtained with physical boundary condition $\phi=\pi$, which are normalized by $\sigma_{0}=\sigma\left(T=0, \mu=0, \mu_{I}=0\right)$; the dual condensates and PL are normalized by their corresponding values at $T=240 \mathrm{MeV}$. We mainly focus on the thermal behaviors of these quantities near the phase transitions. 
Figure. 3 shows that $|\pi(\phi=\pi)|(\Phi)$ decreases (increases) monotonically with $T^{1}$, but $|\sigma(\phi=\pi)|$ first increases (slowly) up to $T \sim 175 \mathrm{MeV}$ and then decreases. These results are also qualitatively agreement with that obtained in [16] within the PNJL model.

Consistent with Fig. 2, Fig. 3 indicates that the normalized DPC really behaves like an order parameter for center symmetry: analogous to the DPL obtained in $[3,8,11]$, it keeps rather small value in low temperature region and gradually becomes larger with $T$. Like the thin PL, the DPC increases monotonically with $T$. However, the normalized DPL in Fig. 3 shows abnormal thermal behavior, which first reduces with $T$ (up to $T \sim 175 \mathrm{MeV}$ ) and then raises. Similar to the PNJL result, we see that the DPL is quite sensitive to the $T$-dependence of the quark condensate rather than the PL near the phase transition region. In addition, the DPL and DPC rise rapidly near the chiral transition and the melting of the pion condensate, respectively. This is also consistent with the PNJL results.

Several susceptibilities defined as the $T$ derivatives of the quantities displayed in Fig. 3 are shown in Fig. 4. As in $[14,16]$, the peak of a susceptibility is used to locate the critical temperature. Fig. 4 indicates that the PL susceptibility has one peak, which indicates $T_{c}^{P}=$ $175 \mathrm{MeV}$. Different from Fig. 5 in [16], the other susceptibilities also only have one peak, which is very close to $T_{c}^{P}$. We see that the highest peak of $\partial \Sigma_{\sigma}{ }^{1} / \partial T$ coincides with that of $\partial|\sigma| / \partial T$, and the corresponding critical temperatures $T_{c}^{d \sigma}$ and $T_{c}^{\sigma}$ are slightly lager than $T_{c}^{P}$. The coincidence of $T_{c}^{d \sigma}$ and $T_{c}^{\sigma}$ is consistent with [14, 16]. In addition, Fig. 4 also shows that the critical temperatures $T_{c}^{d \pi}$ and $T_{c}^{\pi}$ (extracting from $\partial \Sigma_{\pi}{ }^{1} / \partial T$ and $\partial|\pi| / \partial T$, respectively) also coincide, which almost take the same value as $T_{c}^{P}$. So the double peaks of the susceptibilities other than that of the thin PL one observed in PNJL [16] disappear totally due to the enhanced entanglement between the PL and quark and pion condensates in EPNJL model. However, we still notice that there exists a slight difference between $T_{c}^{P}$ and $T_{c}^{d \sigma}$ (or $\left.T_{c}^{d \pi}\right)$.

\section{Conclusion}

The investigation of the thermal behavior of dual condensates at finite isospin chemical potential for $\mu_{I}>m_{\pi}$ has been performed in an entangled PNJL model. Compared to the PNJL model, the entanglement of the center symmetry breaking and the chiral transition or the evaporation of the pion condensate with $T$ is enhanced in EPNJL. We mainly focus on the role of this strengthened entanglement on the thermal properties of the dual observables by considering the pion condensation.

We find that the twisted angle dependences of the quark and pion condensate obtained in EPNJL are still qualitatively consistent with the PNJL results in [16] and the dual pion condensate also exhibits the order parameter like thermal behavior as the thin PL. Due to the competition between the quark and pion condensates, the abnormal thermal behavior of the dressed PL found in [16] is still observed in EPNJL, which suggests that this dual condensate is also quite sensitive to the chiral transition rather than the deconfinement described by the PL. On the other hand, the critical temperatures extracted from the quark and pion condensates and their dual partners are very close to the one determined by the thin PL. This is quite different from the PNJL results.

Anyway, whether the dual observables carry the deconfinement information is an interesting question. Which result of these two models is more close to QCD can be determined

\footnotetext{
${ }^{1}$ This is also observed in [20] and other chiral model studies [21]. The reason for such an anomaly is that the quantity $\sqrt{\sigma^{2}+\pi^{2}}$ always decreases with $T$ but $|\pi|$ drops more quickly near the phase transition since $\lambda$ is zero but $m$ is finite.
} 
by the lattice simulation with physical quark masses, which is free from the sign problem at finite isospin chemical potential.

\section{Acknowledgements}

Z.Z. was supported by the NSFC ( No.11275069).

\section{References}

[1] Y. Aoki, S. Borsanyi, S. Durr, Z. Fodor, S. D. Katz, S. Krieg and K. K. Szabo, JHEP 0906, 088 (2009).

[2] S. Borsanyi et al. [Wuppertal-Budapest Collaboration], JHEP 1009, 073 (2010).

[3] E. Bilgici, F. Bruckmann, C. Gattringer and C. Hagen, Phys. Rev. D 77, 094007 (2008).

[4] E. Bilgici, F. Bruckmann, J. Danzer, C. Gattringer, C. Hagen, E. M. Ilgenfritz and A. Maas, Few Body Syst. 47, 125 (2010).

[5] B. Zhang, F. Bruckmann, C. Gattringer, Z. Fodor and K. K. Szabo, AIP Conf. Proc. 1343, 170 (2011) [arXiv:1012.2314 [hep-lat]].

[6] J. Braun, L. M. Haas, F. Marhauser and J. M. Pawlowski, Phys. Rev. Lett. 106, 022002 (2011).

[7] C. S. Fischer, Phys. Rev. Lett. 103, 052003 (2009).

[8] C. S. Fischer and J. A. Mueller, Phys. Rev. D 80, 074029 (2009).

[9] C. S. Fischer, J. Luecker and J. A. Mueller, Phys. Lett. B 702, 438 (2011).

[10] C. S. Fischer, A. Maas and J. A. Muller, Eur. Phys. J. C 68, 165 (2010).

[11] K. Kashiwa, H. Kouno and M. Yahiro, Phys. Rev. D 80, 117901 (2009).

[12] R. Gatto and M. Ruggieri, Phys. Rev. D 82, 054027 (2010).

[13] T. K. Mukherjee, H. Chen and M. Huang, Phys. Rev. D 82, 034015 (2010).

[14] S. Benic, Phys. Rev. D 88, 077501 (2013).

[15] F. Marquez, A. Ahmad, M. Buballa and A. Raya, Phys. Lett. B 747, 529 (2015) [arXiv:1504.06730 [nucl-th]].

[16] Z. Zhang and Q. Miao, arXiv:1507.07224 [hep-ph].

[17] K. I. Kondo, Phys. Rev. D 82, 065024 (2010) doi:10.1103/PhysRevD.82.065024 [arXiv:1005.0314 [hep-th]].

[18] Y. Sakai, T. Sasaki, H. Kouno and M. Yahiro, Phys. Rev. D 82, 076003 (2010) doi:10.1103/PhysRevD.82.076003 [arXiv:1006.3648 [hep-ph]].

[19] D. T. Son and M. A. Stephanov. Phys. Rev. Lett. 86 , 592 (2001); Phys. At. Nucl. 64, 834 (2001).

[20] Z. Zhang and Y. -X. Liu, Phys. Rev. C 75, 064910 (2007).

[21] L. y. He, M. Jin and P. f. Zhuang, Phys. Rev. D 71, 116001 (2005). 